\title{
MAKER: Stronger Frames by Mimicking Nature
}

\section{Mr. Dustin Jack Hallenbeck, University of Pittsburgh, Johnstown}

Dustin Hallenbeck is a senior, mechanical engineering technology student at the University of Pittsburgh, Johnstown minoring in mathematics. Dustin focuses in innovative product design and advanced manufacturing processes

\section{Dr. Tumkor Serdar, University of Pittsburgh, Johnstown}

Serdar Tumkor is an Assistant Professor of Mechanical Engineering Technology at University of Pittsburgh at Johnstown. Dr. Tumkor has more than 20 years of experience in education, having taught at Stevens Institute of Technology and Istanbul Technical University. His engineering experience includes design, manufacturing, and product development. He has been lecturing Manufacturing Processes, Machine Design, Engineering Design, and Computer Aided Technical Drawing courses. 


\title{
Stronger Frames by Mimicking Nature
}

\begin{abstract}
Quadcopters and first person view drones (FPV) have become extremely popular over the last few years while the prices were decreasing. Quadcopter includes mechanical and electronic components that needs to be designed lightweight. Quadcopter components are made of carbon fiber composite or PE. The purpose of this study is to design a lightweight but stronger quadcopter frame. A nature inspired quadcopter frame has been designed and manufactured with additive manufacturing. A finite element analysis (FEA) is completed before designing the nature inspired frame to see the stress distribution on a solid frame. Actual forces are applied to simulate and see the high stressed regions of the frame, then further modified the frame structure to reinforce these sections by increasing the density of the structure inside the frame. This non-homogenous honeycomb structure resembles the internal structure of a bone. The structure of the frame is engineered to provide higher rigidity while maintaining the light weight benefits. The purpose of this paper is to review the design of quadcopters and to report a lightweight frame design using additive manufacturing. A solid frame is redesigned to maximize its strength to weight ratio by increasing the amount of material in high stress areas, while decreasing material in other areas with lower stress concentrations in order to optimize the lightweight capabilities while maintaining structural integrity.
\end{abstract}

\section{Introduction}

Additive Manufacturing (AM) is still a fairly new manufacturing process. The process starts by taking a $3 \mathrm{D}$ part and breaking it down into $2 \mathrm{D}$ cross sectional areas, while giving a finite thickness ${ }^{1}$. Additive manufacturing provides the opportunity to manufacture integrated components that are difficult to make as a single component with other manufacturing processes. It is predicted to revolutionize the way parts are manufactured, perhaps even on the scale of the industrial revolution with regards to efficiency and innovation ${ }^{2}$. Computer Aided Design (CAD) and Finite Element Analysis (FEA) in unison will allow for virtual design, characterization, and production of scaffolds optimized for the replication of cellular structure in manufactured components ${ }^{3}$. AM has the capability of taking complex assemblies and turning them into single components, making them stronger and cheaper to manufacture in the process. The speed and ease of use for small production runs makes it revolutionarily innovative. As AM is developed and refined, new materials and processing times will be drastically reduced making this process extremely viable. Additive manufacturing has been used for roughly 15 years, which was mostly used for rapid prototyping instead of full-scale manufacturing. Over the past few years, this has been changing and the true potential of AM is being realized. Making components that have internal geometries and can still be a single component will bring entire assemblies into single parts, while strengthening them, and saving money on tooling, materials, and machining ${ }^{4}$. Manufacturing is coming to a point to where the only limitations are the minds of designers and engineers. With these tools, a micro quadcopter frame will be designed with an internal porous frame that will be instilled while still composed of one solid manufactured part that can then be tested and analyzed to discover if this is a viable design and product.

A new trend in the United States for technological hobbies has been quadcopters and first person view quadcopters, also known as micro drones. Micro quadcopters now make it possible for the common hobbyist to be able to build, using selected parts, and fly a quadcopter without spending a large amount of money. Micro quadcopters average between 6 to 8 inches in length from end to 
end, as seen in Figure 1. Currently high end devices are built with a carbon fiber frame which cost roughly $\$ 25-50$ USD to purchase from online retailers ${ }^{5}$.

This study looks at the implication of additive manufacturing for a micro quadcopter frame. The study will maximize a weight to power ratio for an internal skeletal structure of the frame, giving it strength where needed and remove excess material from where it is not. This process was designed to test and analyze the creation of a custom designed micro quadcopter frame. This will be done by maximizing the flight weight of the frame and then conducting a finite element analysis of the frame while under the force of flight. The frame will then be maximized to match the high force points of the frame. This specific design example would have been difficult to manufacture using any other form of manufacturing due to the artificial organic porosity inside of the frame. A structure like this, containing a porous material to strengthen while keeping the component light weight, has been found throughout multitudes of organic material.

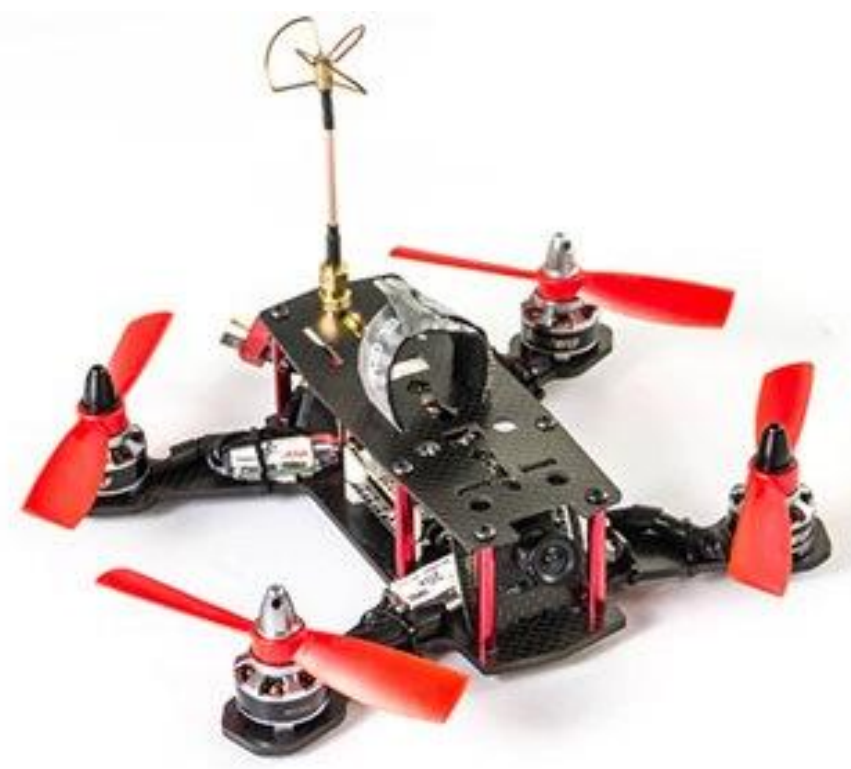

Figure 1 Quadcopter Frame

\section{Equipment and Manufacturing}

The 3D printer selected for this project was a MakerBot Replicator V3 (Figure 2). The ease of access, usability, and accuracy of this machine made it an easy decision for the project. The base model has factory settings making each layer 0.1 millimeters thick. The thickness was changed to the lowest setting for the accuracy of the project, allowing each injected layer of plastic to be at the finite thickness of 0.05 millimeters ${ }^{6}$. With the increase in accuracy came the increase in time, making the run time of the project roughly 19 hours instead of the estimated 6 hours. The increase in time accounts for each printing pass requiring more precision and the number of layers having doubled. 


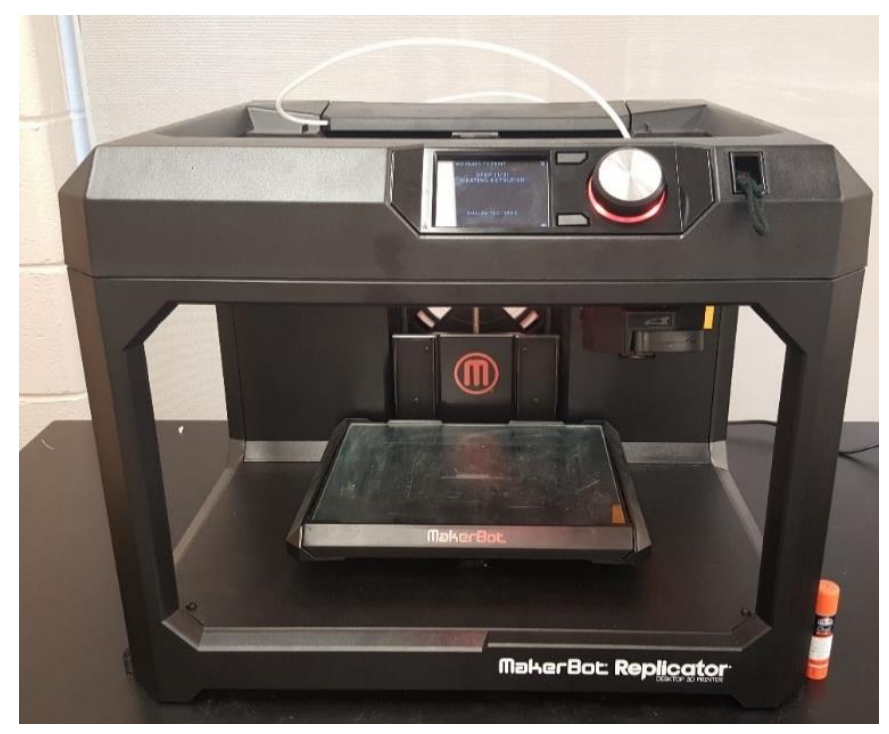

Figure 2 MakerBot Replicator V3

MakerBot also comes equipped with its own custom interface and software. MakerBot software can be downloaded from their website and it allows you to upload multiple different platforms of $3 \mathrm{D}$ models. Once the model is entered into the software it then has to be oriented properly so that the printing can commence. 3D printed polymer structures need support material because the overhang material cannot support itself. MakerBot has the capability to print support material that are needed to be removed after printing. However, the structure of the designed quadcopter frame has the advantage of being produced on a 3D printer without any additional support material.

\section{Quadcopter Frame Design}

The advancements in coding and sensor technology has not only made it possible to nearly fly any object, regardless of shape, but also allows anyone be able to pick up the controller and fly any quadcopter. The processor planned for this project comes equipped with a 3 axis gyroscope that will do automatic leveling and consistent flight patterns ${ }^{7}$. Even with this advance in technology, the frame's balance must still be analyzed carefully, making sure that all components of any major mass, excluding the motors, be as centralized inside the body as possible. The suggested design frame is an entry level racing frame that has a mounting system pre designed making it possible to have a micro camera mounted and turning the quadcopter into a first person view (FPV) or drone. All components have been centralized and the weight distribution has been taken into account. The most noticeable weight is the battery inside the unit, which is mounted on top and will be held down by a strap going through the slide rail. This makes it possible to rebalance the quadcopter if a camera or other accessories are added so that it can still be fairly balanced and adjusted between each flight at the owner's discretion, as shown in Figure 3. 


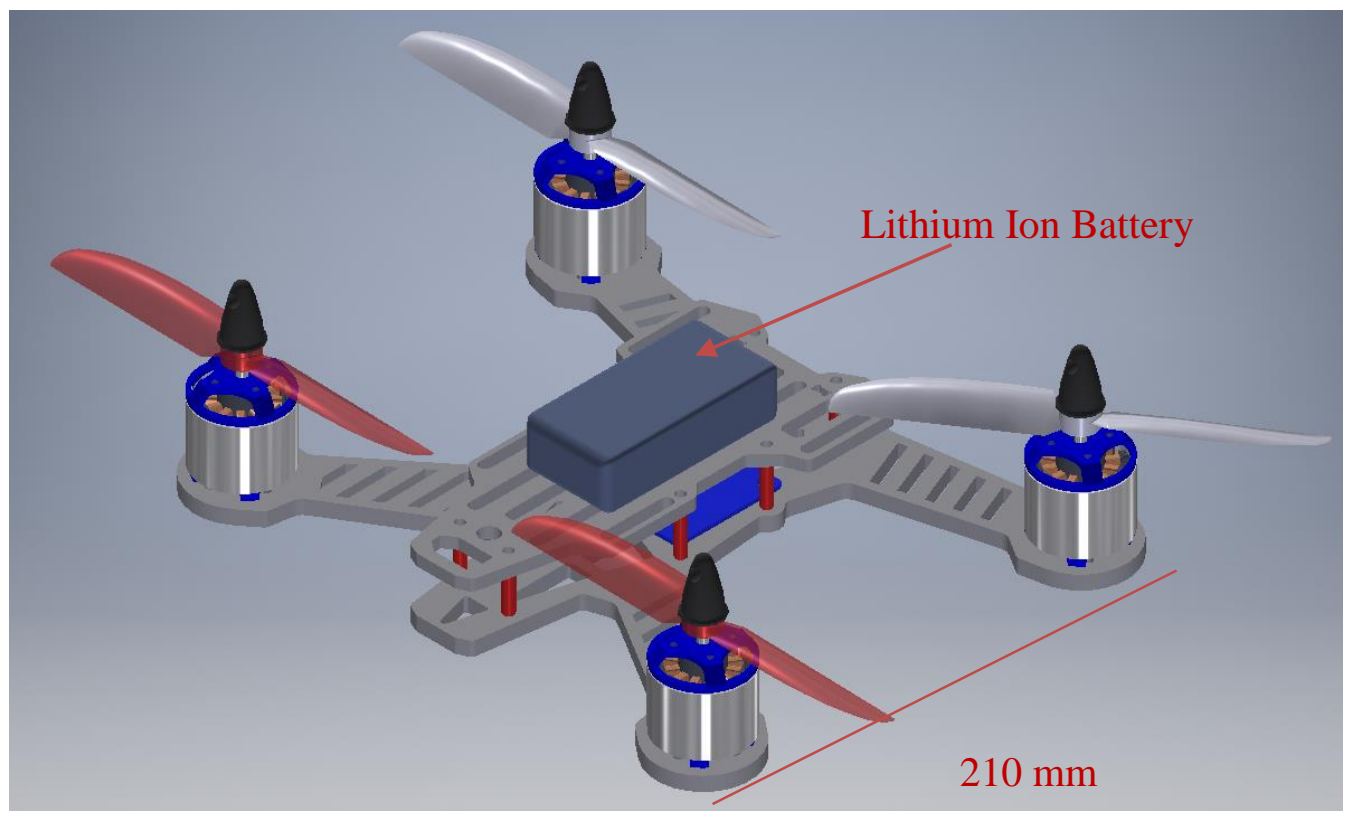

Figure 3 Quadcopter Assembly

The redesign objective was to decrease the amount of material on the frame as much as possible without decreasing the rigidity of the frame. The frame shown in Figure 3 is a 210 millimeter long frame. It has been adjusted from the original size to accommodate larger components and have the ability to be modified into an FPV. The larger the quadcopter, the larger the motors and propellers must be, giving more force and the ability to carry heavier loads and larger batteries. The external shell of the frame is entirely solid like any common quadcopter frame. The inside of the frame has a porous structure throughout that reduces material usage while strengthening the frame (Figure 4). At this point the internal frame can be combined with a set of parametric equations which can then add more supports where necessary, depending on stress concentrations throughout the frame.

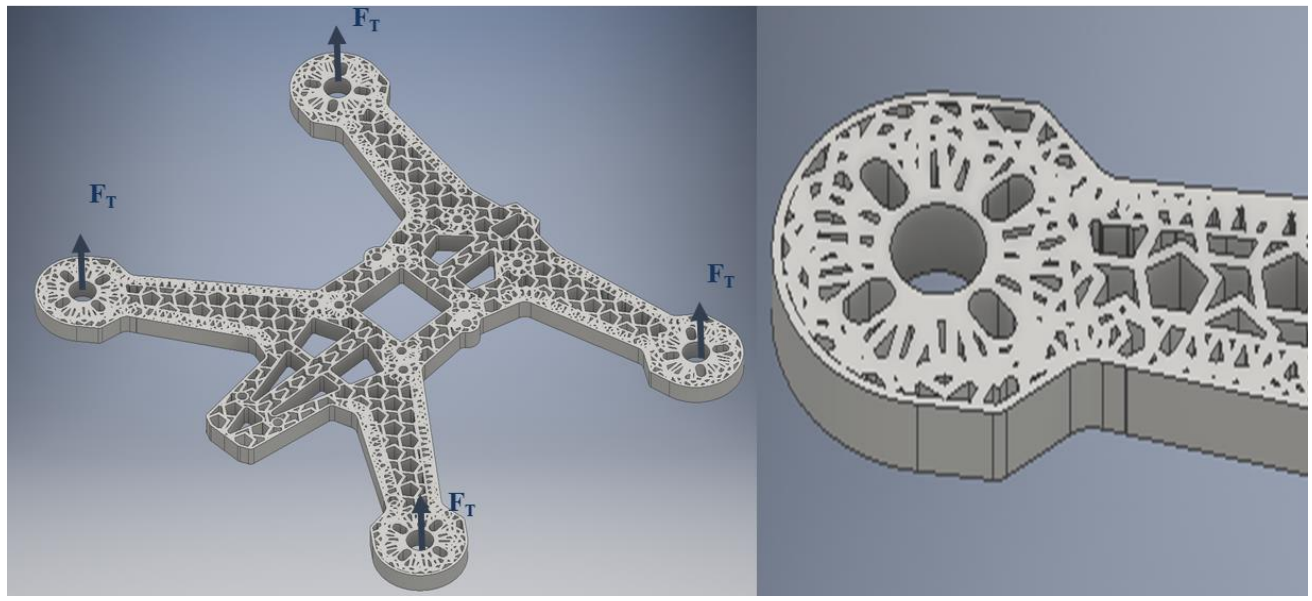

Figure 4 Internal Structure of the Frame 


\section{Porous Structure and Manufacturing}

Crashes are common when dealing with micro quadcopters, so impact resilience is a high priority. Designing a small space between each layer of plastic gives the product flexibility during a hard impact, such as falling out of the sky. The frame of the quadcopter is manufactured with an additive manufacturing process. The frame of the quadcopter is given a much more natural and porous shape, making it both lightweight and stronger in areas where the plastic needs to be reinforced, such as the frame arms, and hollow in sections where minimal impact can be expected, like the center plate of the frame. This concept of cellular structure within parts can be seen through nature fairly frequently. Bones are perfect examples (Figure 5) as the structural system allows the bone to flex slightly when taking an impact and has also been proven to be very strong, while maintaining a lightweight characteristic. This study attempted to recreate the combination of cancellous bone tissue, also known as sponge bone tissue, internally with an external rigid frame of cortical bone tissue ${ }^{8}$ in a manufactured product to reduce material costs and reduce stress concentrations. Synthetic bones are being made and tested using additive manufacturing in the same process as this design. The product is then more impact resistant because it will flex and move slightly with the openings in these cells, while the current non-cellular structure is more brittle and more likely to crack and fracture on heavier impacts. This idea is used between every layer of plastic. The organic design has the potential to maximize the flexible rational design, through control of the architecture and functionalization of the pores in multiple areas in the design ${ }^{9}$. All of these reasons combine to make the quadcopter frame an optimal component for additive manufacturing with polymers. With additive manufacturing processes advancing so quickly, the option of testing multiple different materials along with multiple structures is also a big opportunity for additive manufacturing while using the same piece of equipment. Options for different materials range from polymers and plastics, to metals.

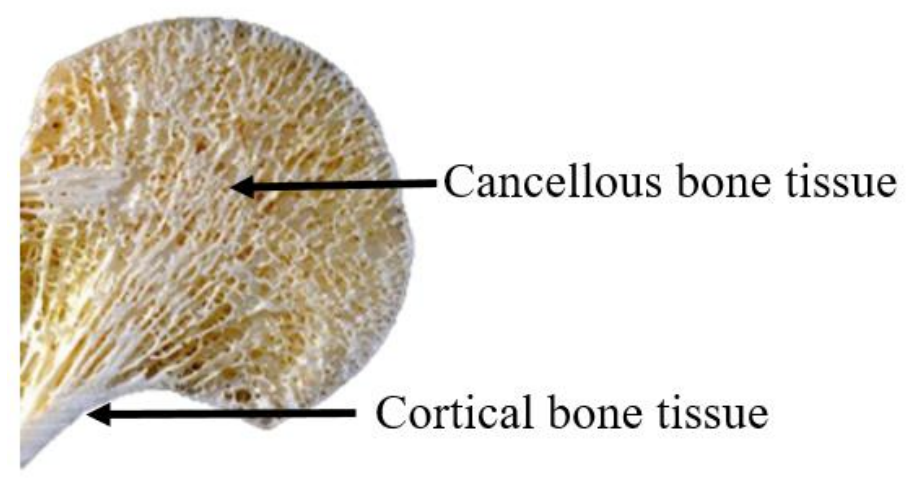

Figure 5 Mesostructured bone

Once the structure was designed and tested through a printing simulation, it was time to begin the printing process. First, the printer began to make a raft for the print to stick to and be removed from the printer once the printing process is completed, as shown in Figure 6. 


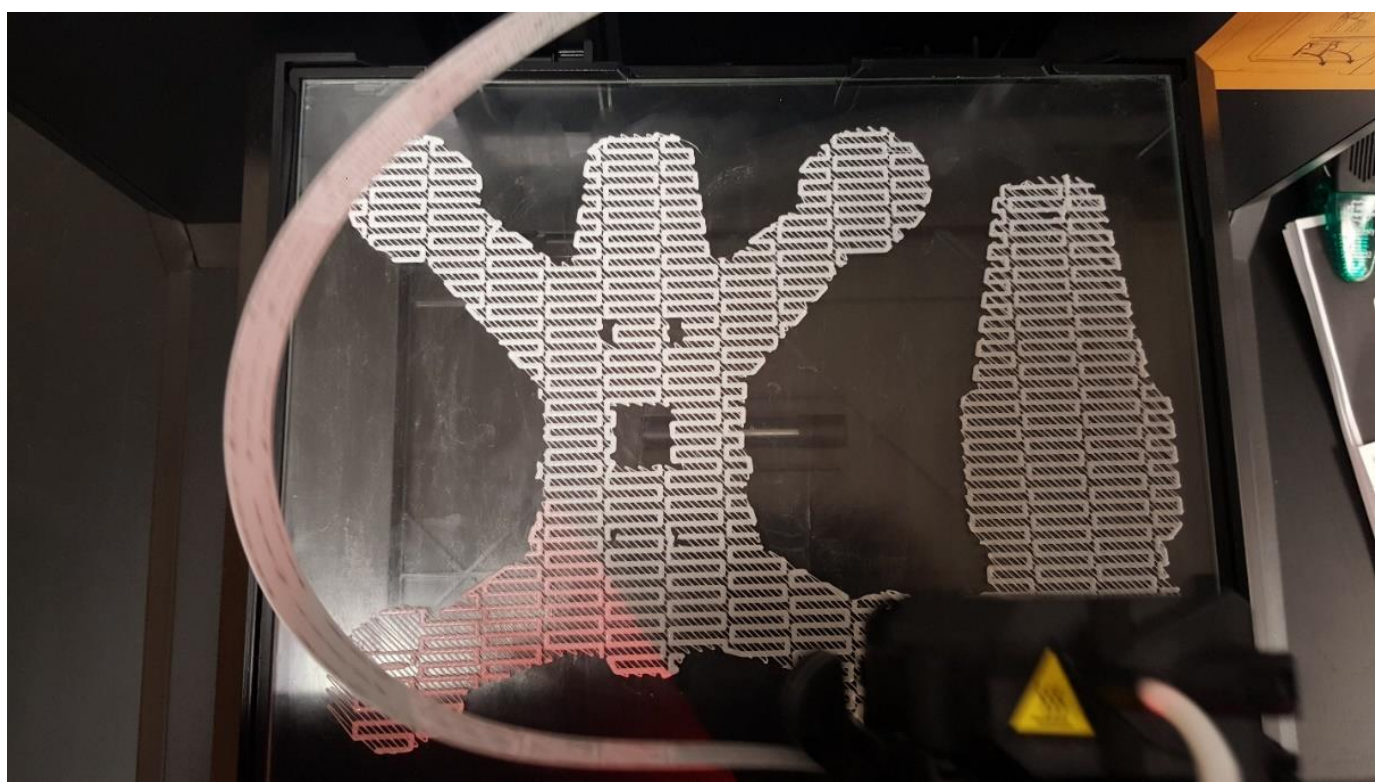

Figure 6 Initial Raft

The product is then printed and laid on top of the raft. At this point the product is checked on frequently to make sure that it is printing properly. If no issues appear then the tray that holds the component will lower and give access to the finalized product shown in Figure 7.

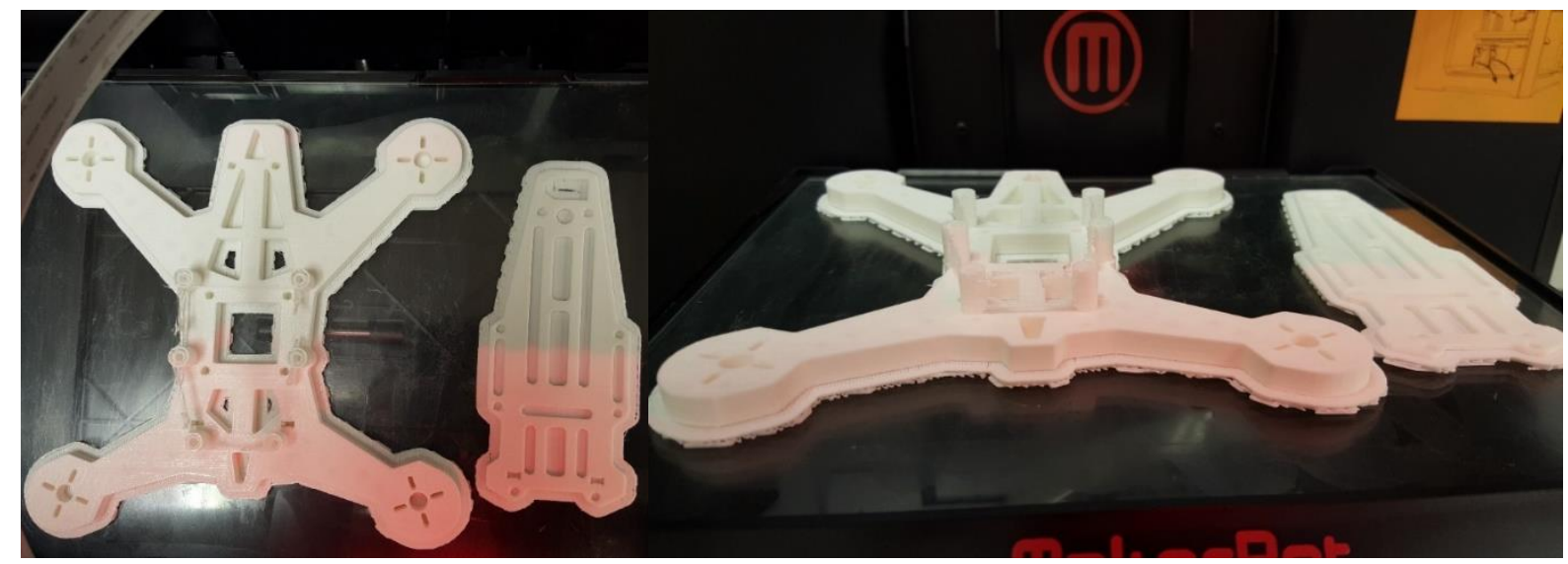

Figure 7 Finalized Print

If multiple components have been made they can be assembled using a light epoxy as shown in Figure 8. 


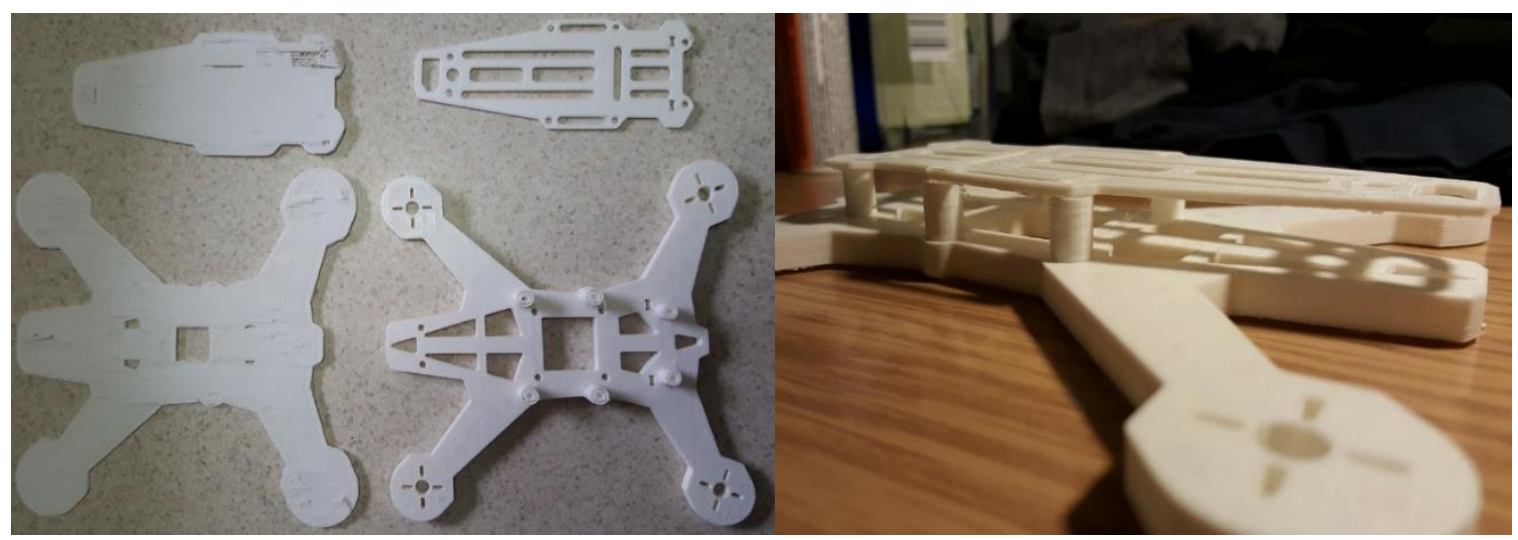

Figure 8 Raft removal and assembled final product

\section{Analysis}

The boundary conditions of this static structural analysis are shown in the wire frame in Figure 9, along with the condition of limiting the points where the motor mounts to only allow changes in the Y-direction. These simulations were designed for the condition of constant flight under normal weight conditions. After analysis was completed the variations in frame design can be shown in Figure 9. The first variation in frame was a simple vent pattern along the motor mount arms. This was successful in removal of excess material, however gave high stress concentrations at the corners of each vent hole. Next variation was the simple pentagonal pattern, or honeycomb pattern. While this design was the most successful when it came to reduction of material, it had enough material in certain areas but was lacking in others. To combat the lack of material, the final iteration was the bone structured frame. While building off the general pentagonal pattern, material was now added in known high stress areas. This design helped spread the stress evenly throughout the frame, while still keeping the material reduction to the maximum allowable. The choice of a pentagonal pattern was selected for the high bending strength of this shape, making it more rigid than a shape like a square or hexagon that has multiple points of symmetry ${ }^{3}$. Taking advantage of parametric modeling the frame can now be maximized for a strength to weight ratio. Looking at the flight simulations will give a clear point as to where the high pressure zones of the frame are and can be adjusted accordingly to deal with these pressure points ${ }^{10}$. The final pattern gave a reduction in material of nearly $25 \%$ while still keeping a rigid frame that is well within yield criteria.

Using a general pentagon shape in known high stress points, the number of pentagons can then be increased, allowing them to overlap and increase the amount of the material in these areas. Joints and connection points can then have an increased volume when high induced stress situations occur. Now that each of these patterns is made, it is extremely easy to input the amount of force that will be applied to the frame within the simulation to create an increase in the volume using the overlapping pentagonal pattern that mimics the bone structure. Maximizing this parametric set can be adapted to any additive manufacturing project, even if it is to be used as the fill layers for the software used to convert drawings into the printing files creating a parametric mesostructure. 


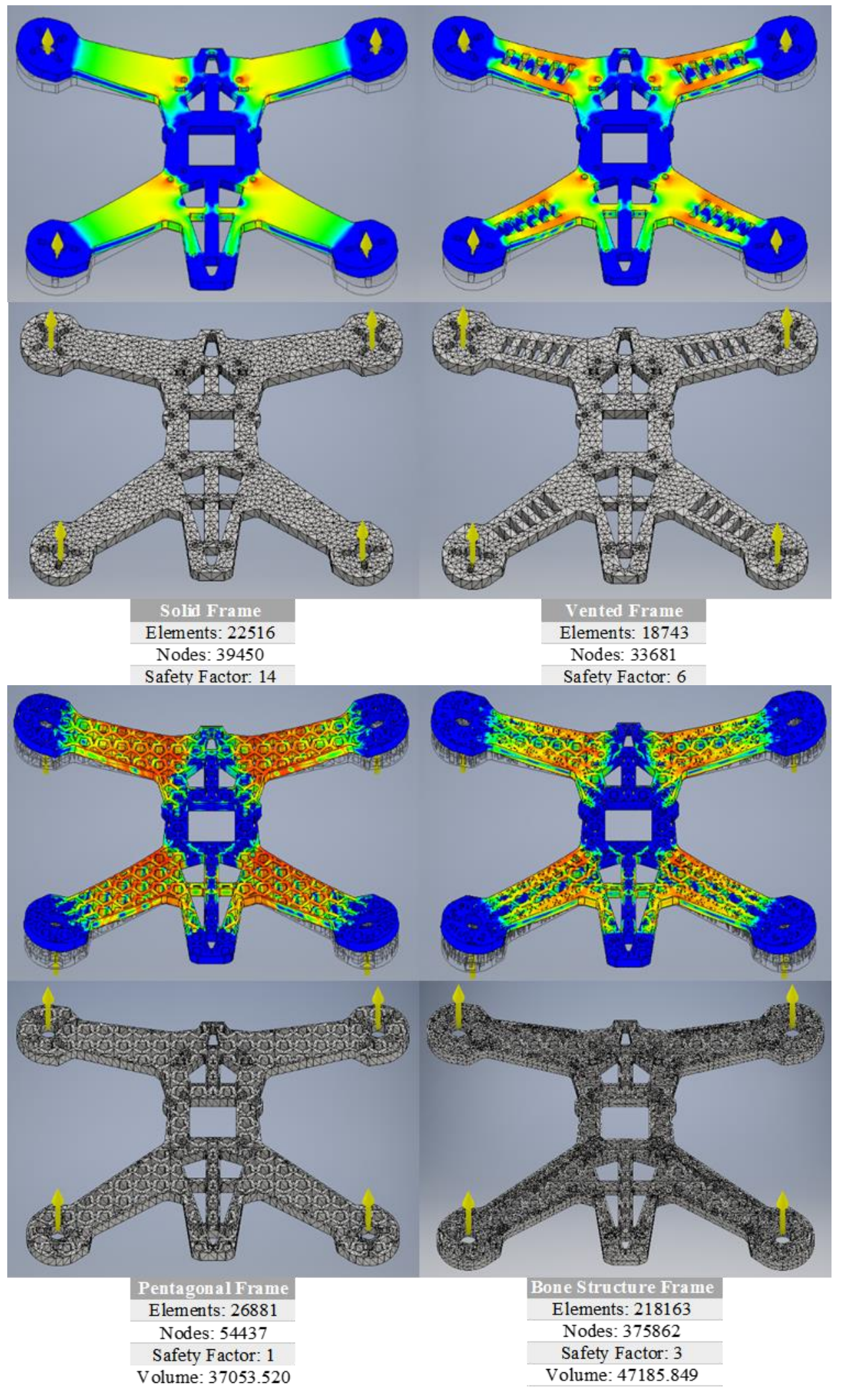

Figure 9 Analysis steps of each frame design 


\section{Conclusion}

The combination of parametric design and AM will make the prototyping process extremely quick while making the testing of multiple examples of products much more cost effective and efficient. For this specific case, it gives the customer the opportunity to test multiple different frames and replace broken frames easily and for a fraction of the cost, allowing for exploration and innovation within the field of quadcopters and drones. Having a porous internal structure similar to many types of natural cell structures gives the quadcopter frame an excellent balance of both rigidity and ductility during flight and impact. This complex design can be produced without any additional cost using additive manufacturing. Internal frame design can be further altered with the implication of a parametrically defined CAD models. The parametric model permits easy adjustments for different material properties, different loads, boundary conditions, and variations in shape and size of the internal pattern. Mimicking natural structures will maximize the material efficiency, while removing excess material from locations that have a minimal risk of failure. The finalized design keeps rigidity that will withstand flight conditions, while removing nearly $25 \%$ of excess material. Removing a $25 \%$ cost in materials for manufacturing could greatly decrease the cost of these parts. With the ability of additive manufacturing in the home, this product could also be varied and tested for different situations and load cases by the customer themselves. The same approach can be implemented to a multitude of different applications including, but not limited to, sporting equipment, car parts, air craft components, household equipment, furniture, and many more.

\section{Acknowledgments}

This project is being carried out at the University of Pittsburgh at Johnstown. The research idea has been granted by the UPJ College Council in 2016. This support is gratefully acknowledged.

\section{References}

[1] De Borst, R., Crisfield, M. A., Remmers, J. J., \& Verhoosel, C. V. (2012).Nonlinear finite element analysis of solids and structures. John Wiley \& Sons.

[2] Berman, B. (2012). 3-D printing: The new industrial revolution. Business horizons, 55(2), 155-162.

[3] Wettergreen, M. A., Bucklen, B. S., Liebschner, M. A. K., \& Sun, W. (2008). CAD assembly process for bone replacement scaffolds in computer-aided tissue engineering. In Virtual Prototyping \& Bio Manufacturing in Medical Applications (pp. 87-111). Springer US.

[4] Kruth, J. P., Leu, M. C., \& Nakagawa, T. (1998). Progress in additive manufacturing and rapid prototyping. CIRP Annals-Manufacturing Technology, 47(2), 525-540.

[5] ZMR180 Mini Quad ARF, Retrieved from http://www.fpvmodel.com/zmr180-mini-quadarf-pro-w-t-motor-motors-and-littlebee-escs_g1073.html

[6] MakerBot Replicator Desktop 3D Printer. (n.d.). Retrieved March 11, 2016, from http://www.pcmag.com/article2/0,2817,2491092,00.asp

[7] Nemati, A., \& Kumar, M. (2014, June). Modeling and control of a single axis tilting quadcopter. InAmerican Control Conference (ACC), 2014 (pp. 3077-3082). IEEE.

[8] Gibson, L. J., \& Ashby, M. F. (1999). Cellular solids: structure and properties. Cambridge university press. 
[9] Li, H., Eddaoudi, M., O'Keeffe, M., \& Yaghi, O. M. (1999). Design and synthesis of an exceptionally stable and highly porous metal-organic framework.Nature, 402(6759), 276279.

[10] Rosen, D. W. (2007). Computer-aided design for additive manufacturing of cellular structures. Computer-Aided Design and Applications, 4(5), 585-594. 